\title{
Relation between Depression and Hormonal Dysregulation
}

\author{
Neiane de Souza Duarte, Lucas Maciel de Almeida Corrêa, Larissa Rodrigues Assunção, \\ Arthur Aguiar de Menezes, Onássis Boeri de Castro, Leda Fabiélen Teixeira
}

Department of Medicine at Faculdades Integradas Aparício Carvalho-FIMCA, Porto Velho, Rondônia, Brazil

Email: neiane_duarte@hotmail.com

How to cite this paper: de Souza Duarte, N., de Almeida Corrêa, L. M., Assunção, L. R., de Menezes, A. A., de Castro, O. B., \& Teixeira, L. F. (2017). Relation between Depression and Hormonal Dysregulation. Open Journal of Depression, 6, 69-78. https://doi.org/10.4236/ojd.2017.63005

Received: May 25, 2017

Accepted: July 28, 2017

Published: July 31, 2017

Copyright ( 92017 by authors and Scientific Research Publishing Inc. This work is licensed under the Creative Commons Attribution International License (CC BY 4.0). http://creativecommons.org/licenses/by/4.0/

\section{Open Access}

\begin{abstract}
Depression is a mental disorder, with different classifications which have as main symptoms, persistent sadness, lack of interest, lack of pleasure, swings between feelings of guilt and low self-esteem, sleep disorders and appetite. It is known that hormonal changes can lead to significant emotional changes and vice versa, due to changes in the central nervous system, action of hormones on specific receptors or by metabolic changes, therefore, the endocrine disorders become one of the possible causes of depression. This work is a study of the major hormones associated with depression. It was drawn from a literature review, in which corticotrophin hormone, cortisol, estrogen, progesterone and thyroid hormones were identified as the main hormones related to depression. These hormones are essential for the correct functioning of the metabolism, therefore, it is observed that hormonal changes may contribute to the development of depression as well as aggravate it or even hamper the treatment of patients who already have the disorder.
\end{abstract}

\section{Keywords}

Depression, Hormones, Endocrine Disorders

\section{Introduction}

World Health Organization (WHO) defines depression as a common mental disorder, which, similar to the other depressive disturbances is characterized by persistent sadness, lack of interest and pleasure, fluctuations between feelings of guilt and low self-esteem, as well as sleep and/or appetite disorders that affect the everyday operation. These manifestations occur in the different types of depression and are distinguished in terms of intensity, duration and mode of presentation (WHO, 2017). Major depression, from mild to moderate, is characterized by 
episodes of depressive symptoms and some functional disabilities. Severe depression is accompanied by agitation and psychomotor retardation, as well as marked somatic symptoms, frequently observed in chronic diseases (APA, 2014). In addition to these factors, depression is more prevalent in females. According to a study carried out in 23 European countries, the depressive disorder is twice higher in women compared to men, reaching them mainly in the fertile periods (Velde et al., 2010). This enhances the relation between hormonal variation and depression, since this is more common in females who undergo constant hormonal influences due to the menstrual cycle. Evidence suggests that hormonal changes may favor the onset of depression, since hormones may be directly associated with the activation, inhibition, or modulation of central nervous system mechanisms associated with depression.

It is known, for example, that around half of the depressed patients present an increase in the reactivity of the hypothalamic-pituitary-adrenal (HPA) axis, represented by the increase of $\mathrm{CRH}$ (corticotrophin releasing hormone) (Varghese et al., 2001). This state of hormonal imbalance can lead to numerous physiological changes, which would explain a common series of consequences to the depressive syndrome, such as metabolic changes.

Seeman (1997) emphasize that estrogens, notably estradiol, play an active role in the organization of brain development and play a crucial role in maintaining brain activity. They also protect neurons from developmental dysfunctions (Schizophrenia) and degenerative dysfunctions (Alzheimer's disease). Cyclic fluctuations in estrogen and progesterone increase stress responses, which make them susceptible to depression and anxiety.

It is also observed that acutely depressed patients present an increase in free T4 in the cerebrospinal fluid (CSF), which reverts with the improvement in the condition (Kirkegaard et al., 1990), and low serum levels of T3 are associated with a more rapid recurrence of depressive episodes (Jackson, 1998; Dayan et al., 2013). Low levels of free thyroid hormones and elevated levels of thyroid stimulating hormone (TSH), even within normal levels, are associated with delayed therapeutic response in bipolar depression (Price et al., 2007). Disorders of thyroid function are clearly related to the evolution and response to antidepressant treatment, and it is often necessary to replace T4 in hypothyroidism, or potentiation with T3, in resistance to antidepressant treatment (Teng et al., 2005).

Based on evidence found in studies linking hormonal dysregulation and depression, a detailed description of the relation of major hormones associated with depression will be given below.

\section{Corticotrophin and Cortisol in Depression}

Corticotropin-releasing hormone $(\mathrm{CRH})$ is responsible for the regulation of Adrenocorticotropic hormone (ACTH) release, which is related to the hypothalamic-pituitary-adrenal (HPA) axis and associated with neurotransmission and mediation in autonomic, immunological, neuroendocrine and cardiovascular responses. Its release is induced by the production of corticotrophin-releasing 
hormone (CRH), secreted by the neurons of the hypothalamic paraventricular nucleus in the portal circulation of the pituitary gland. In the anterior pituitary, CRH stimulates ACTH secreting cells to release ACTH into the bloodstream. ACTH acts on the adrenal cortex promoting the release of Cortisol into the bloodstream (Graeff, 2007).

$\mathrm{CRH}$ promotes the secretion of adrenocorticotrophic hormone (ACTH) through the pituitary, leading to the release of cortisol (involved with stress and adrenaline release), estrogen and mineralocorticoids. Some depressive and anxiety disorders are associated with increased corticoid production (cortisol), while melancholic depression is in turn linked to increased CRH in the cerebrospinal fluid (CSF) (Ayala, 2002).

CRH is the mainstay in the HPA axis and is essentially involved in the pathophysiology of stress and consequently of depression, since many patients report its onset after stressful events. In this sense, the pathophysiology of stress is similar to the major depression, where in both cases there is decreased appetite, sleep disturbances and hypervigilance. It is believed that reduced libido and psychomotor changes in depression are associated with the release of this hormone (Juruena et al., 2004). Major depression, in turn, is associated with the abnormal release of CRH and deregulation of the HPA axis, consequently, increased release of ACTH, in addition to excessive secretion of the corticosteroid cortisol (hypercotisolemia). Depressed patients have altered amounts of CRH mRNA and protein in the paraventricular nucleus (PVN) of the hypothalamus, in addition to the increased amount of corticotrophin in the cerebrospinal fluid (Graeff, 2007). A phenomenon linked to increased secretion of this hormone is the alteration of REM sleep (Chellappa \& Araújo, 2006), which becomes fragmented, where the decrease of "latency" to REM sleep is a persistent event in depressive conditions. Other elements, such as the phenomena of maternal deprivation and social isolation are triggers of depression, culminating in hyperactivation of the HPA axis and the production of CRH.

In addition, during pregnancy, there is a large production of $\mathrm{CRH}$, which is cut shortly after delivery. It may help to develop postpartum depression associated with the hormone concentration in the plasma (increased in the third trimester of pregnancy). Ayala (Ayala, 2002) explains that " $\mathrm{CRH}$ circulates in plasma in minimal amounts, except in the last trimester of pregnancy, when there are considerable increases in serum levels."

In an attempt to improve depressive symptoms, note whether CRH-selective antagonist as a potential treatment option. Drugs that influence the production and reception of corticosteroids are also being studied. Researchers have concluded that there are antidepressants that have direct action on glucocorticoid receptors, although their function is also linked to neurotransmitters and cytokines (Juruena et al., 2004).

\section{Depression and Female Sexual Hormones}

It is believed that women have a greater tendency to develop depression due to 
drastic hormonal changes in puberty, in each menstrual cycle, in the postpartum period, and in menopause. In the middle of uncertainties, evidence of depression in women has pointed to sex hormones, because they affect mood, cognition, and mental status (Li et al., 2005). Estrogen and progesterone are the ovarian steroid hormones, responsible for the sexual development of the woman and the menstrual cycle.

In recent years, more and more evidence has indicated that estrogen plays key roles in the central nervous system. Recent research shows that not only affects the hypothalamus, but also hippocampus and cerebellum, where most cognitive functions, motor skills and mood are controlled (Justo et al., 2010).

Arpels (1996) state that "the brain in women, has been shown to be an estrogen target organ." Its presence in the central nervous system is related to the growth and differentiation of dendrites and axons in the developing brain, dendrites arborization and synaptogenesis, in the production and release of neurotransmitters and as a protector against glutamatergic cytotoxicity ( $\mathrm{Li}$ et al., 2005).

At the beginning of puberty, the woman starts the production of gonadal hormones, acquiring the so-called "periodicity". This fact, coupled with the onset of abrupt hormonal changes, seems to contribute to the development of mood disorders (Veras et al., 2005). These hormones influence the psychic state of the adolescent by the modulation of the serotoninergic system (Amin et al., 2005).

In addition to the typical hormonal changes of puberty, there are psychosocial stresses that help increase the predisposition of the depressive disorder. Body changes occur at about the same time that is seen the high rate of depression, indicating a considerable probability of connection between the altered body image, physical and hormonal changes and the onset of depression in puberty (Maj et al., 2005).

A comparative study between institutionalized patients and controls from 7 to 17 years indicated that the serum level of 5-HT is reduced in patients with mood disorder. In addition, the fact that there is a better response to treatment with serotonergic medications, when compared to noradrenergic action, also corroborates the hypothesis. Therefore, the circulating hormones produced by the gonads modulate brain neurotransmission and have a role in the regulation of mood states (Scivoletto et al., 2009).

There is strong evidence that serotonin is mediating the effects of estrogens on mood modulation and cognition. Studies in humans, particularly performed on menopausal women who undergo estrogen therapy, have provided subsidies for these assertions and identified situations in which mood and cognition changes are accompanied by changes in serotonergic function and hormonal status (Amin et al., 2005).

Estrogen has action in the central nervous system (CNS), even in regions that have no action on the reproductive cycle. It has a trophic effect on cholinergic neurons and significantly stimulates the expression of serotonergic type $2 \mathrm{~A}$ re- 
ceptors in areas that regulate both mood and cognition. The ability of estrogen to act as a serotonergic agonist modulator is due not only to the increased expression of 5HT-2A receptors, but also through increased serotonin synthesis, prolactin response to serotonergic agonists and monoamine oxidase (MAO) degradation and reduction of the 5HT-1 binding sites and the serotonin transporter messenger ribonucleic acid (mRNA). All these actions have a similar antidepressant effect (Pecins-Thompson et al., 1998).

In contrast to estrogens, progesterone acts to increase MAO activity, resulting in a decrease in serotonin levels and a depressive effect. In animal model studies using labeled hormone, progesterone receptors were demonstrated in cells of the medial hypothalamus and in the cells of the middle eminence region, cortex, amygdala, hippocampus and coeruleous locus (Bloom, 1988). These are areas associated with emotional reactions, behavior, learning and endocrine regulation. In these areas, progesterone produces depressant effects on those functions. It also presents anesthetic, tranquilizing, mood stabilizing properties, with reduced mental activities, and has anticonvulsive action. It would therefore have opposite effects on estrogen on the CNS (Silva et al., 2006).

\section{Depression and Thyroid Hormones}

In a survey of 43 individuals diagnosed with depression (19 women and 24 men) serum levels of thyroid hormones were tested (Berent et al., 2014). T3 was tested in patients, where 13 patients were identified with low levels and 30 with normal levels. Among the patients with low T3 levels, 4 had low levels of T4, and 24 had normal levels of this hormone. Among patients with normal levels of T3, 3 presented low levels of T4. Considering all the values according to the gender of the patient.

It has been observed that the reduction of thyroid hormones is directly linked to the decrease in serotonin serum levels (Cleare et al., 1995). Bahls (1999) states that serotonin is a neurotransmitter heavily involved with depression. When researchers have investigated the relation between the serotoninergic system with the thyroid hormone function, it was observed a decrease in the response of prolactin and cortisol to 5-HT agonist d-fenfluramine in hypothyroid patients not treated, which leads to believe 5-HT (5-hydroxytryptamine) decreases in these cases. When sequenced in the investigation, they ratified what they had observed, when they found that serum serotonin levels normalized with the replacement of thyroid hormones. They concluded that in hypothyroid cases, serotonergic neurotransmission may decrease, and this condition may be reversed with the replacement of hormones decline (Cleare et al., 1996).

As a justification for this considerable relationship between thyroid hormones and serotonin, there is the effect of hormones on serotonergic autoreceptors. The presynaptic 5-HT-1A receptors act detecting the presence of serotonin and coupling to them by interrupting the flow of neuronal impulses and consequently leading to a decrease in serotonin release. In depression there is an in- 
crease in the number of these receptors, so there is an increased uptake of serotonin. The decrease in these autoreceptors results in the release of cortical and hippocampal serotonin (Heal et al., 1998). A study with euthyroid rats ( $p=$ 0.011) (Gur et al., 1999) evidenced a significant loss of 5HT-1A autoinhibitory sensitivity following T3 hormone administration. Studies (Bauer et al., 2002; Whybrow et al., 1981) state that thyroid hormones reduce the sensitivity of 5-HT-1A autoreceptors. The results (Altshuler et al., 2001) indicate that the use of T3 may then increase the cortical release of 5-HT.

The interaction of serotonin with thyroid hormone dysfunctions can also be explained when related to the metabolizing enzymes of thyroid hormones (Kirkegaard et al., 1998). Assuming that intracerebral T3 is a result of the deiodination of T4 by deiodinase II (D-II), it is observed the increased enzymatic activity of D-II in hypothyroidism and decreased in hyperthyroidism.

If D-II increases T3 production, and therefore elevates local production of serotonin (Kirkegaard et al., 1998), in contrast, type III deiodinase (D-III) is shown to be increased in hyperthyroidism and decreased in hypothyroidism. It is assumed that the D-III decreases production of T3 and indirectly serotonin production. As we have seen, both hyperthyroidism and hypothyroidism have the altered functioning of these enzymes, which metabolize thyroid hormones and thus can modify brain levels of serotonin. The use of desipramine (Campos-Barros et al., 1994) and fluoxetine (Baumgartner et al., 1994) in rats showed increased D-II activity, and fluoxetine decreased the D-III. Thus, an increase of T3 and of cerebral serotonin occurring, is demonstrated the performance of these drugs as regulators of the neurotransmitter in cases of depression.

We can observe another perspective for the relation between thyroid hormones and depression, from the point that serotonin seems to inhibit TRH (thyrotropin releasing hormone) (Morley, 1981) and in cases of depressed patients, where serotonin is reduced, there is an increase in TRH as well as a stimulus in TSH (thyroid stimulating hormone) secretion. This increase in TSH leads to an increase in the production of $\mathrm{T} 3$ and $\mathrm{T} 4$, which by feedback tends to reduce the serum TSH level, and thus there is a new plasma balance due to an increased T4 concentration (Kirkegaard et al., 1990).

Another possible etiology for depression that is also related to thyroid hormones is the lack of catecholamine, noradrenaline (NE) (Bahls, 1999). Rozanov \& Dratman (1996) found increased concentrations of T3 in the locus coerulus and the lateral tegumentary nucleus of the rat brain, reinforcing studies (Mason et al., 1993) that show higher concentrations of T3 in synaptosomes located in the nuclei of noradrenergic neurotransmission (Dratman et al., 1987). Thus, it has been suggested that T3 plays a special role in noradrenergic nuclei (Rozanov et al., 1996): a role that regulates neurotransmission, which is important when related to thyroid hormones, since noradrenaline is essential in the deiodination of T4 into brain T3. In a 1969 study, they stated (Prange et al., 1969) that T3 caused an increase in the sensitivity of noradrenergic brain receptors. As shown in studies (Rozanov et al., 1996) T3 is largely concentrated in regions of nora- 
drenergic neurotransmission, thus being shown to be a co-transmitter of cerebral noradrenaline.

T3 supposedly acts on beta-adrenergic post synaptic receptors (Whybrown et al., 1981), a similar action with the antidepressant drugs, which explains why the T3 is effective in antidepressant therapy in euthyroid individuals. It was therefore concluded that thyroid hormones help in recovery from depression by increasing beta-adrenergic receptor function. Noradrenaline also participates in the stimulation of the release of TRH and TSH (Morley, 1981). Therefore, a reduction in these hormones may result in a decrease in beta-adrenergic receptors, and thus a decrease in noradrenergic neurotransmission. These adrenergic alterations occur in thyroid diseases. In a study with lymphocytes from animals and patients with changes in thyroid hormones, it was observed that beta-adrenergic receptors were increased in cases of hyperthyroidism and decreased in cases of hypothyroidism (Bilezikian et al., 1983; Fregly et al., 1975). For Linnoila et al. (1983), a deficient concentration of brain T3 may lead to an inversion of the amount of adrenergic receptors, where alpha-adrenergics will predominate over beta-adrenergics. Although further evidence is needed on these findings, Howland (1993) states that studies, even in animals, support the beta-adrenergic neurotransmitter system in the pathogenesis of depression.

\section{Conclusion}

In summary, the main hormones associated with depression mentioned in this article were: corticotrophin, cortisol, estrogen, progesterone and thyroid hormones. The analyzed bibliographies demonstrated the relation of these hormones in the regulation of the central nervous system, through the control of specific receptors and metabolic intermediates. This makes it possible to associate depression with hormonal dysregulation. High rates of cortisol, for example, combined with depressive behaviors may be the first biological marker of depression. In an antagonistic way, the estrogen has antidepressive action improving the state of humor, while progesterone is linked to the decrease of serotonin generating depressive effect. These hormones alter the production of serotonin $(5-\mathrm{HT})$ receptors, a neurotransmitter highly related to depression. In the same way, thyroid hormones act. Thus, in addition to reduce the sensitivity of serotonin, autoreceptors are also related to depression due to the alterations of the metabolizing enzymes of these hormones, which can contribute to the development of depression, as well as aggravate it or even make it difficult to treat those who already have the disorder. Hormones, therefore, may be directly associated with the activation, inhibition or modulation of central nervous system mechanisms and consequent depression.

\section{References}

Altshuler, L. L., Bauer, M., Frye, M., Gitlin, M. J., Mintz, J., Szuba, M. P., Leight, K. L., \& Whybrow, P.C. (2001). Does Thyroid Supplementation Accelerate Tricyclic Antidepressant Response? A Review and Meta-Analysis of the Literature. American Journal of 
Psychiatry, 158, 1617-1622. https://doi.org/10.1176/appi.ajp.158.10.1617

Amin, Z., Canli, T., \& Epperson, C. N. (2005). Effect of Estrogen-Serotonin Interactions on Mood and Cognition. Behavioral and Cognitive Neuroscience Review, 4, 43-58. https://doi.org/10.1177/1534582305277152

Arpels, J. C. (1996). The Female Brain Hypoestrogenic Continuum from the Premenstrual Syndrome to Menopause. A Hypothesis and Review of Supporting Data. Journal of Reproductive Medicine, 41, 633-639.

Ayala, A. R. (2002). Corticotrophin Releasing Hormone Antagonists: Update and Perspectives. Arq Bras Endocrinol Metab, 46.

Bahls, S. (1999). Depression: A Brief Review of the Biological and Cognitive Foundations. Rev Interação, 3, 49-60.

Bauer, M., Baur, H., Berghofer, A., Strohle, A., et al. (2002). Effects of Supraphysiological Thyroxine Administration in Healthy Controls and Patients with Depressive Disorders. Journal of Affective Disorders, 68, 285-294. https://doi.org/10.1016/S0165-0327(00)00363-3

Baumgartner, A., Dubeyko, M., Campos-Barros, A., Eravci, M., \& Meinhold, H. (1994). Subchronic Administration on Fluoxetine to Rats Affects Triiodothyronine Production and Deiodination in Regions of the Cortex and in the Limbic Forebrain. Brain Research, 635, 68-74. https://doi.org/10.1016/0006-8993(94)91424-9

Berent, D., Zboralski, K., Orzechowska, A., \& Gałecki, P. (2014). Thyroid Hormones Association with Depression Severity and Clinical Outcome in Patients with Major Depressive Disorder. Molecular Biology Reports, 2419-2425.

https://doi.org/10.1007/s11033-014-3097-6

Bilezikian, J., \& Loeb, J. (1983). The Influence of Hyperthyroidism and Hypothyroidism on Alfa- and Beta-Adrenergic Receptor Systems and Adrenergic Responsiveness. Endocrine Reviews, 4, 378-388. https://doi.org/10.1210/edrv-4-4-378

Bloom, F. E. (1988). Neurotransmitters: Past, Present, and Future Directions. The FASEB Journal, 2, 32-41.

Campos-Barros, A., Meinhold, H., Stula, M., Müller, F., Köhler, R., Eravci, M., Putzien, O., \& Baumgartner, A. (1994). The Influence of Desipramine on Thyroid Hormone Metabolism in Rat Brain. Journal of Pharmacology and Experimental Therapeutics, 268, 1143-1152.

Chellappa, S., \& Araújo, J. (2006). Sleep and Sleep Disorders in Depression. Journal of the Federal University of Rio Grande do Norte, 1-5.

Cleare, A., McGregor, A., \& O’Keane, V. (1995). Neuroendocrine Evidence for an Association between Hypothyroidism, Reduced Central 5-HT Activity and Depression. Clinical Endocrinology, 43, 713-719.

Cleare, A., McGregor, A., Chambers, S., Dawling, S., \& O’Keane, V. (1996). Thyroxine Replacement Increases Central 5-Hydroxytryptamine Activity and Reduces Depressive Symptoms in Hypothyroidism. Clinical Endocrinology, 64, 65-69.

Dayan, C. M., \& Panicker (2013). Hypothyroidism and Depression. European Thyroid Journal, 2, 168-179.

Dratman, M., Crutchfield, F., Futaesaku, Y., Goldbereger, M., \& Murray, M. (1987). Triiodothyronine in the Rat Brain: Evidence for Neural Localization and Axonal Transport Derived from Thaw-Mount Film Autoradiography. Journal of Comparative Neurology, 260, 392-408.

Fregly, M., Nelson, E., Resch, G., Field, F., \& Lutherer, L. (1975). Reduced Beta-Adrenergic Responsiveness in Hypothyroid Rats. American Journal of Physiology, 229, $916-$ 
924.

Graeff, F. (2007). Anxiety, Panic, and the Hypothalamic-Pituitary-Adrenal Axis. Brazilian Journal of Psychiatry, 3-6.

Gur, E., Lerer, B., \& Newman, M. (1999). Chronic Clomipramine and Triiodothyronine Increase Serotonin Levels in Rat Frontal Cortex in Vivo: Relationship to Serotonin Autoreceptor Activity. Journal of Pharmacology and Experimental Therapeutics, 288, 81-87.

Heal, D., \& Smith, S. (1998). The Effects of Acute and Repeated Administration of T3 to Mice on 5-HT1 and 5-HT2 Function in the Brain and Its Influence on the Actions of Repeated Eletroconvulsive Shock. Neuropharmacology, 27, 1239-1248.

Howland, R. (1993). Thyroid Dysfunction in Refractory Depression: Implications for Pathophysiology and Treatment. Journal of Clinical Psychiatry, 54, 47-54.

Jackson, I. M. (1998). The Thyroid Axis and Depression. Thyroid, 8, 951-956.

Juruena, M., Claire, A., \& Pariante, C. (2004). The Hypothalamic-Pituitary-Adrenal Axis, the Role of Glucocorticoid Receptors and Their Importance in Depression. Brazilian Journal of Psychiatry, 189-201.

Justo, L., \& Calil, H. M. (2010). Depression, the Same Affect for Men and Women? Revista de Psiquiatria Clínica, 74-79.

Kirkegaard, C., \& Faber, J. (1998). The Role of Thyroid Hormones in Depression. European Journal of Endocrinology, 138, 1-9.

Kirkegaard, C., Korner, A., \& Faber, J. (1990). Increased Production of Thyroxine and Inappropriately Elevated Serum Thyrotropin Levels in Endogenous Depression. Biological Psychiatry, 27, 472-476.

Li, R., \& Shen, Y. (2005). Estrogen and Brain: Synthesis, Function and Diseases. Frontiers in Bioscience, 10, 257-267.

Linnoila, M., Cowdry, R., Lamberg, B., Makinen, T., \& Rubinow, D. (1983). CSF Triiodothyronine (rT3) Levels in Patients with Affective Disorders. Biological Psychiatry, $18,1489-1492$.

Maj, M., \& Sartotius, N. (2005). Depressive Disorders. Porto Alegre: Artmed.

Mason, G., Walker, C., \& Prange, A. (1993). L-Triiodothyronine: Is This Peripheral Hormone a Central Neurotransmitter? Neuropsychopharmacology, 8, 253-258.

Morley, J. E. (1981). Neuroendocrine Control of Thyrotropin Secretion. Endocrine Research, 2, 396-436.

Pecins-Thompson, M., Brown, N., \& Bethea, C. (1998). Regulation of Serotonin Reuptake Transporter mRNA Expression by Ovarian Steroids in Rhesus Macaques. Molecular Brain Research, 53, 120-129.

Prange, A., Wilson, I., Rabon, A., \& Lipton, M. (1969). Enhancement of Imipramine Antidepressant Activity by Thyroid Hormone. The American Journal of Psychiatry, 126, 457-469.

Price, B. R., Hatcher, S., \& Korff, V. (2007). Depression in Adults: Psychological Treatments and Care Pathways. Clinical Evidence, $8,1016$.

Rosa, S., Ana Carolina, J. S., \& da Silva, M. F. (2006). Effects of Sex Steroids on Mood and Cognition. Journal of Clinical Psychiatry, 33.

Rozanov, C., \& Dratman, M. (1996). Evidence for Selective Localization of Triiodothyronine in Central Noradrenergic Systems. Neuroscience, 74, 897-915.

Scivoletto, S., \& Tarelho, L. (2009). Depression in Childhood and Adolescence. Brazilian Journal of Medicine, 555-558. 
Seeman, M. (1997). Psychopathology in Women and Men: Focus on Female Hormones. The American Journal of Psychiatry, 154, 1641-1647.

Teng, C. T., Humes, E. C., \& Demetrio, F. N. (2005). Depression and Clinical Comorbidities. Revista de Psiquiatria Clínica, 32, 149-159.

Varghese, F., \& Brown, E. S. (2001). The Hypothalamic-Pituitary-Adrenal Axis in Major Depressive Disorder: A Brief Primer for Primary Care Physicians. Primary Care Companion to the Journal of Clinical Psychiatry, 3, 151-155.

Velde, S. V., Bracke, P., \& Levecque, K. (2010). Gender Differences in Depression in 23 European Countries. Cross-National Variation in the Gender Gap in Depression. Social Science and Medical Journal, 71, 305-313.

Veras, A. B., Nardi, \& Antonio, B. (2005). Female Sexual Hormones and Mood Disorders. Brazilian Journal of Psychiatry.

Whybrow, P. C., \& Prange, A. J. (1981). A Hypothesis of Thyroid-Catecholamine-Receptor Interaction. Its Relevance to Affective-Illness. Archives of General Psychiatry, $38,106-113$

Submit or recommend next manuscript to SCIRP and we will provide best service for you:

Accepting pre-submission inquiries through Email, Facebook, LinkedIn, Twitter, etc. A wide selection of journals (inclusive of 9 subjects, more than 200 journals)

Providing 24-hour high-quality service

User-friendly online submission system

Fair and swift peer-review system

Efficient typesetting and proofreading procedure

Display of the result of downloads and visits, as well as the number of cited articles

Maximum dissemination of your research work

Submit your manuscript at: http://papersubmission.scirorg/

Or contact ojd@scirorg 